\title{
Alterações da densidade mineral óssea em vértebras de ratos Wistar submetidos à ausência de carga nos membros pélvicos
}

\author{
Changes of bone mineral density in vertebrae of rats subjected to hindlimb unload
Eduardo ANTONIETTO ${ }^{1}$; Juliana de Carvalho Apolinário COÊLHO²; Bruna Gabriele BIFFE²; Roberto CARVALHAL ${ }^{1}$; Mário Jefferson Quirino LOUZADA ${ }^{1}$

\author{
${ }^{1}$ Departamento de Apoio, Produção e Saúde Animal da Universidade Estadual Paulista, Araçatuba - SP, Brasil \\ ${ }^{2}$ Departamento de Ciências Básicas da Faculdade de Odontologia da Universidade Estadual Paulista, Araçatuba - SP, Brasil
}

\begin{abstract}
Resumo
A suspensão de ratos pela cauda é modelo utilizado para investigar o comportamento ósseo em animais impossibilitados de se locomoverem. O osso é um tecido adaptativo que se desenvolve em sua estrutura e função, entre outros fatores, em resposta a forças mecânicas aplicadas a ele e demandas metabólicas que venha sofrer. A ausência de forças mecânicas e de deformação óssea faz com que ocorra uma diminuição na deposição de cálcio por ausência de estímulos nos osteoblastos e osteócitos, favorecendo a ação dos osteoclastos, tornando o osso enfraquecido e quebradiço. Portanto, a ação mecânica é necessária para estimular a resposta óssea local e, assim, proporcionar seu crescimento e remodelamento. O objetivo deste estudo foi avaliar, através da densitometria radiográfica, se a suspensão pela cauda por 15 e 36 dias altera a densidade mineral óssea das vértebras cervical $\left(\mathrm{C}_{3}\right)$, torácica $\left(\mathrm{T}_{6}\right)$ e lombares $\left(\mathrm{L}_{1} \mathrm{e} \mathrm{L}_{3}\right)$ de ratos Wistar. Trinta Rattus norvegicus albinus, adultos, machos, linhagem Wistar, massa corpórea média de $\pm 350 \mathrm{~g}$, foram divididos em 3 grupos: controle ( $\mathrm{n}=10)$ - não suspenso; $S_{15}(n=10)$ - suspenso por 15 dias e $S_{36}(n=10)$ - suspenso por 36 dias. Para análise densitométrica, as vértebras foram radiografadas, escaneadas, digitalizadas e analisadas pelo programa computacional ImageJ". Houve aumento estatisticamente significante da densidade mineral óssea no grupo $\mathrm{S}_{15}$, provavelmente pela inquietação dos animais à suspensão, com diminuição no grupo $\mathrm{S}_{36}$, fato este hipoteticamente ligado à acomodação dos mesmos, concluindo que a suspensão pela cauda alterou a densidade mineral óssea num primeiro momento com diminuição com o passar do tempo.
\end{abstract}

Palavras-chave: Densitometria radiográfica. Coluna vertebral. Ratos. Ausência de carga.

\begin{abstract}
The suspension of rats by the tail model is used to investigate the behavior of bone in animals unable to move around. Bone is an adaptative tissue that develops in structure and function, among other factors, in response to mechanical forces applied to it and metabolic demands that it will suffer. The absence of mechanical forces and deformation of bone that occurs causes a decrease in calcium deposition in the absence of stimuli on osteoblasts and osteocytes, favoring the action of osteoclasts, making bones weak and brittle. Therefore, the mechanical action is necessary to stimulate local bone response and thus provide growth and remodeling. The aim of this study was to evaluable by radiographic densitometry, the tail suspension for 15 and 36 days alter the bone mineral density of cervical vertebrae (C3), thoracic (T6) and lumbar (L1 and L3) of Wistar rats. Thirty Rattus norvegicus albinus, adult, male, Wistar strain, average body mass $\pm 350 \mathrm{~g}$, were divided into 3 groups: control $(n=10)$ - not suspended; $S_{15}(n=10)$ - suspended for 15 days and $S_{36}(n=10)$ - suspended for 36 days. For densitometric analysis vertebrae were radiographed, scanned, digitized and analyzed by the computer program ImageJ. There was a statistically significant increase in bone mineral density in group $S_{15}$, probably by the restlessness of the animals to the suspension, with a decrease in group $\mathrm{S}_{36}$, and this hypothetically is linked to the accommodation of the rats, concluding that the tail suspension altered bone mineral density in first time with a decrease over time.
\end{abstract}

Keywords: Radiographic densitometry. Spinal column. Rats. Unload.

\section{Introdução}

A suspensão de ratos pela cauda é um dos modelos utilizados para investigar o comportamento ósseo em animais impossibilitados de se locomoverem. Esta suspensão também serve para simular a ausência de
Correspondência para:

Mario J. Q. Louzada

Departamento de Apoio, Produção e Saúde Animal - Universidade

Estadual Paulista

Rua Clóvis Pestana, n 793, Jd. D. Amélia

Araçatuba, SP, CEP: 16050-680

Tel.: +55(18)3636-1359 Fax:+55(18)3636-1352

E-mail: louzada@fmva.unesp.br

Recebido: 27/04/2011

Aprovado: 27/06/2012

Braz. J. Vet. Res. Anim. Sci., São Paulo, v. 49, n. 3, p. 185-192, 2012 
carga sofrida por indivíduos quando em ambiente de "microgravidade"1,2,3,4.

Como o osso fornece sustentação interna para todo o corpo e inserção para músculos e tendões, suas características mecânicas dependem diretamente da carga a ele submetida ${ }^{5}$.

Por se tratar de um tecido adaptativo, o tecido ósseo, diante de forças mecânicas aplicadas a ele e das demandas metabólicas, desenvolve sua estrutura e função. Quanto maior for a solicitação imposta ao osso pela ativação de osteoblastos, maior será a sua resposta, tornando-o mais resistente, ao passo que, a ausência da solicitação óssea torna-o enfraquecido. Portanto, a resposta óssea local está diretamente ligada ao estímulo da ação mecânica que irá estimular o seu crescimento e remodelamento ${ }^{6}$.

Em estudo prévio, realizado pelos autores deste trabalho no Laboratório de Biofísica da UNESP/ Araçatuba, foram analisadas as alterações mecânicas e densitométricas na coluna vertebral de ratos submetidos à suspensão pela cauda e foram observadas diminuição dos valores da força máxima admitida e rigidez nas vértebras dos animais que foram suspensos. Contudo, a análise da densidade mineral óssea, realizada com o equipamento DPX-ALPHA, não foi suficientemente sensível para determinar a densidade na região de interesse, principalmente devido às pequenas dimensões das vértebras dos ratos, mesmo o equipamento possuindo um programa especial para análise em pequenos animais. Com relação à questão metodológica para a avaliação da densidade mineral óssea em peças de pequena dimensão ${ }^{7}$, considerou a densitometria radiográfica como ferramenta capaz de avaliar estas peças se utilizados programas de processamentos de imagens.

A diminuição da massa óssea não ocorre uniformemente em todo o corpo, mas é mais acentuada em determinadas regiões como na coluna vertebral, provavelmente, devido à diminuição das atividades diárias $^{8,9}$, podendo gerar enfraquecimento ósseo, decor- rente de doenças osteometabólicas, como a osteoporose. A osteoporose é caracterizada pela diminuição da densidade mineral óssea, com deterioração da microarquitetura óssea, levando a um aumento da fragilidade esquelética ${ }^{10}$. Durante a ausência de carga no esqueleto, mesmo em curtos períodos, pode ocorrer o enfraquecimento ósseo ${ }^{11}$, assim foi proposta deste estudo utilizar a densitometria radiográfica e o programa computacional de processamento de imagens "Image J" como método de avaliação da densidade mineral óssea das vértebras cervical $\left(\mathrm{C}_{3}\right)$, torácica $\left(\mathrm{T}_{6}\right)$ e lombares $\left(\mathrm{L}_{1}\right.$ e $\left.\mathrm{L}_{3}\right)$ de ratos que foram suspensos pela cauda por 15 e 36 dias.

\section{Material e Método}

A constituição dos grupos experimentais foi dada por 30 ratos, adultos, machos, espécie Rattus novergicus albinus, linhagem Wistar, com massa corpórea de 350 gramas em média, divididos em grupo controle (C), grupo suspenso pela cauda por 15 dias $\left(\mathrm{S}_{15}\right)$ e grupo suspenso pela cauda por 36 dias $\left(\mathrm{S}_{36}\right)$. Adotou-se o método de suspensão preconizado em trabalhos anteriores neste trabalho ${ }^{3,4,12}$. Os animais dos grupos suspensos permaneceram durante todo o experimento com os membros torácicos apoiados no chão da gaiola.

Após o período de experimento de cada grupo, os animais foram eutanasiados e todo o segmento vertebral foi isolado. Para isso, foram retiradas a pele, as inserções musculares e a medula espinhal. Feito isso, todo o segmento vertebral foi imerso em água e deixado em repouso, por três meses, sob abrigo da luz, para que ocorresse desintegração da matéria orgânica por microorganismos formados durante o processo.

Após três meses, as vértebras foram retiradas do recipiente e escovadas com detergente para a retirada de qualquer fragmento orgânico e elementos gordurosos que ainda existissem. Logo após, os ossos foram submetidos, por três dias, à imersão em solução de 
água oxigenada 20 volumes para clareação. A seguir, as vértebras foram armazenadas em freezer à temperatura de $-20^{\circ} \mathrm{C}$. Foi eleita para análise a $3^{\mathrm{a}}$ vértebra cervical, a $6^{\mathrm{a}}$ torácica e a $1^{\mathrm{a}}$ e $3^{\mathrm{a}}$ lombares; vértebras estas, exceto a $1^{\text {a }}$ lombar, por estarem localizadas na metade dos segmentos cervical, torácico e lombar, respectivamente.

Foram feitas tomadas radiográficas das vértebras em análise as quais foram digitalizadas com resolução 200 DPI, em scanner HP - HP Scanjet G4050 acoplado a adaptador para transparências da mesma marca, e as imagens digitalizadas foram armazenadas com extensão TIFF (Figura 1).

Como referencial densitométrico nas tomadas radiográficas, foi utilizada escada de alumínio (liga6063, ABNT) de nove degraus, colocada próxima às vértebras, na direção do feixe principal dos Raios X ${ }^{13,14}$.

O programa computacional "Image J" - versão 1.43u (domínio público - http://rbs.info.nih.gov/ij) foi utilizado para contornar toda região de interesse, utilizando recurso de definição de área do progra- ma, e, assim, determinar o nível de densidade média, em tons de cinza (até 256 níveis), das vértebras e dos degraus da escada de alumínio. Os valores das vértebras foram convertidos para valores relativos à espessura em milímetros de alumínio ${ }^{13,14}$, obedecendo as etapas descritas abaixo por Louzada ${ }^{7}$. Desta maneira, a densidade óssea foi expressa em equivalente de alumínio (mmAl).

Os valores de densidade radiográfica foram convertidos para valores de espessura com o auxílio do computador e, assim, a densidade passou a ser expressa em equivalentes de milímetros de alumínio. Para tanto, os dados foram levados à planilha Excel e passaram pelas seguintes etapas: 1) Determinou-se o valor da Densidade Radiográfica (DR) da Região de Interesse (RDI); 2) Utilizando-se como referência o valor de DR da etapa 1, definiu-se 3 degraus da escada de alumínio, que foram usados como referência densitométrica, cujos valores de DR's contemplavam o da etapa $1 ; 3$ ) Com os valores de espessura e DR dos 3 degraus da etapa 2, construiu-se um gráfico com a planilha Excel; 4) Com ferramentas do Excel, definiu-se

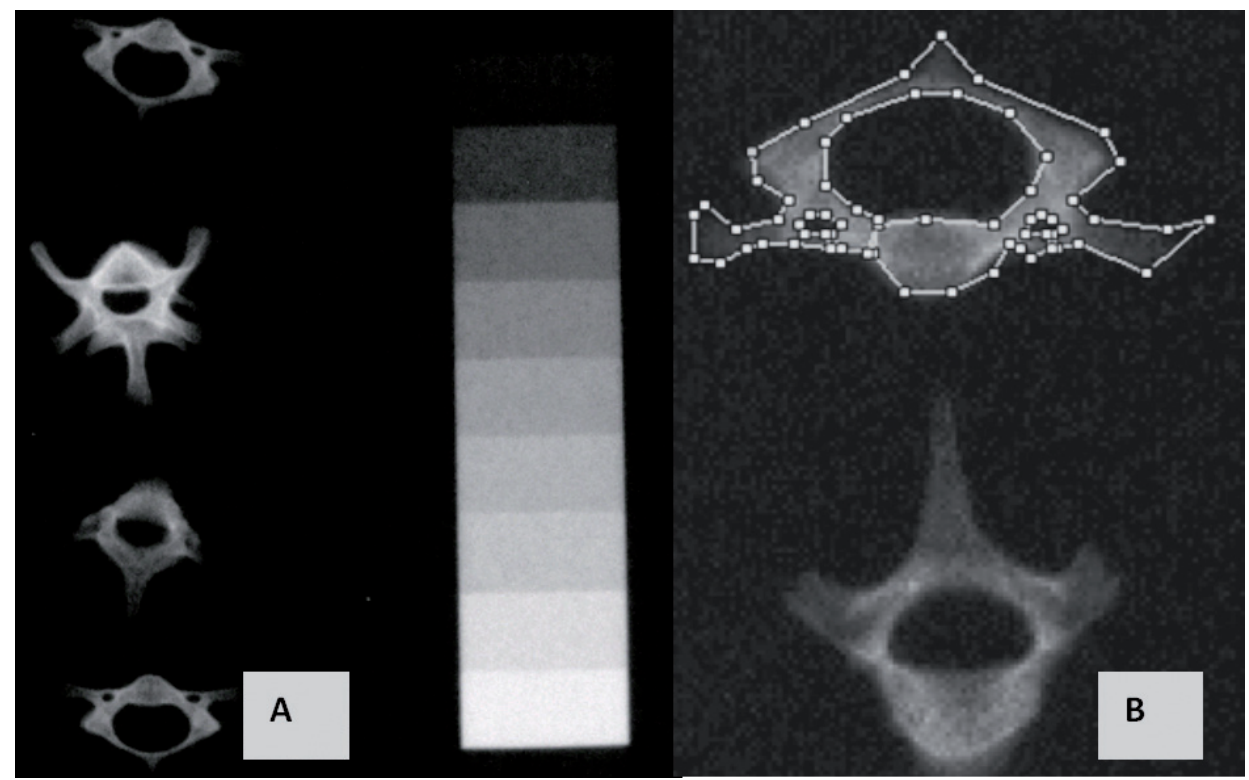

Figura 1 - Imagem da tela do computador com o programa “Image J". Em A, tem-se as vértebras e a escada de alumínio, utilizada como referencial densitométrico e em B, a Região de Interesse circundada na $4^{\mathrm{a}}$ vértebra cervical, utilizando ferramentas do programa Image J 
a melhor curva que se ajustava aos pontos do gráfico (polinômio do $3^{\circ}$ grau). O programa forneceu a equação de ajuste; 5) Utilizando-se a equação de ajuste da etapa 4, fez-se a substituição do valor de X pela DR da RDI (etapa 1) e determinou-se assim a espessura equivalente em mmAl para aquele objeto em estudo ${ }^{7}$.

Para a análise estatística foi utilizado o programa Graphpad Instat 3. Foi realizado análise de variância - ANOVA - para os valores de densidade óssea dos grupos - C, $\mathrm{S}_{15}$ e $\mathrm{S}_{36}$ - e teste de Tukey, com nível de significância $5 \%$.

\section{Resultados}

Os resultados de densidade mineral óssea - DMO, em mmAl, das vértebras dos animais dos três grupos de estudo estão apresentados na tabela 1. De todas as vértebras dos animais estudados, foram excluídas três vértebras por quebrarem durante o processo de armazenamento.

Em análise separada dos grupos, observou-se que a densidade óssea dos animais do grupo $\mathrm{C}$ é maior na $3^{\text {a }}$ e $1^{\text {a }}$ lombar, seguida da $3^{\text {a }}$ cervical e $6^{a}$ torácica respectivamente (Tabela 1 ).

No grupo $S_{15}$, observou-se permanência de maior densidade mineral óssea na $3^{\mathrm{a}} \mathrm{L}$, seguida pela $1^{\mathrm{a}} \mathrm{L}$ e aproximação dos valores da $6^{\mathrm{a}} \mathrm{T}$ em relação à $3^{\mathrm{a}} \mathrm{C}$. $\mathrm{O}$ grupo $\mathrm{S}_{36}$ apresentou maior densidade óssea na $3^{\mathrm{a}} \mathrm{L}$, seguida pela $1^{\mathrm{a}} \mathrm{L}, 3^{\mathrm{a}} \mathrm{C}$ e $6^{\mathrm{a}} \mathrm{T}$, sendo que esta última apresentou valor muito próximo ao do grupo $\mathrm{C}$. $\mathrm{Na}$ figura 2 estão representados os valores da densidade mineral óssea (DMO) dos grupos $\mathrm{C}, \mathrm{S}_{15}$ e $\mathrm{S}_{36}$, respectivamente.

Quando confrontadas as vértebras do mesmo segmento entre os grupos, a $\mathrm{DMO}$ da $3^{\mathrm{a}} \mathrm{C}$ e da $6^{\mathrm{a}} \mathrm{T}$ não apresentou diferença significante $(\mathrm{p}>0,05)$ entre os grupos, figuras 3 e 4 , respectivamente. Ao comparar a DMO das vértebras L1 e L3 entre os grupos, observase que o grupo $S_{15}$ apresenta maior densidade mineral

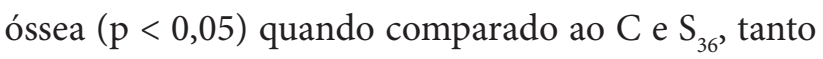
para L1 (Figura 5) como para L3 (Figura 6).
A análise das vértebras do grupo $\mathrm{C}$, que permaneceu solto na gaiola, mostrou que a maior densidade mineral óssea se concentrou em L3, seguida respectivamente por L1, C3 e T6.

\section{Discussão}

O tecido ósseo é um tecido adaptativo ${ }^{6}$ e a suspensão pela cauda por 21 dias prejudica a manutenção das propriedades ósseas ${ }^{15}$, pois um ambiente desprovido de estímulos mecânicos produz efeitos diretos na estrutura óssea alterando sua função ${ }^{16,17}$, e a exposição a ambientes de microgravidade e também a não deambulação afetam diretamente o sistema músculoesquelético, o que gera uma baixa tensão sobre os ossos e, por consequência, menor massa óssea seria necessária para manter a integridade estrutural do esqueleto $^{18}$, assim, neste experimento, esperava-se que a densidade mineral óssea dos animais dos grupos $\mathrm{S}_{15}$ e $\mathrm{S}_{36}$ diminuíssem em relação ao grupo $\mathrm{C}$ ao final do mesmo, entretanto, foi observado um comportamento inesperado dos referidos valores, em que o grupo $\mathrm{S}_{15}$ obteve um aumento nos valores da densidade mineral óssea das vértebras em estudo em relação ao grupo controle. A ordem de densidade mineral óssea das vértebras ficou distribuída na mesma sequência que o grupo $\mathrm{C}$, onde $\mathrm{L} 3>\mathrm{L} 1>\mathrm{C} 3>\mathrm{T} 6$. Tal acontecimento pode ser atribuído devido ao fato dos membros pélvicos terem ficado livres e móveis, mesmo com a suspensão pela cauda e devido à inquietação dos animais diante do posicionamento, o que os levou a uma constante contração dos músculos do tronco, em especial, do dorso, na tentativa de se livrar da suspensão. Muitos músculos do dorso do rato, como o músculo serrátil posterior superior e o músculo serrátil posterior inferior, estão inseridos nas vértebras da coluna e diante da contração dos mesmos, as vértebras acabam sofrendo estimulação favorecendo a osteogênese local ${ }^{19}$.

Em decorrência dos valores apresentados no grupo $\mathrm{S}_{15}$, e sabendo que a conservação do metabolismo 
Tabela 1 - Densidade Mineral Óssea, em mmAl, das vértebras (3a Cervical, $6^{\text {a }}$ Torácica, $1^{\text {a e }} 3^{\text {a }}$ Lombares) dos animais do Grupo C, $\mathrm{S}_{15}$ e S $_{36}$ - Araçatuba - SP, 2010

\begin{tabular}{|c|c|c|c|c|c|c|c|c|c|c|c|c|}
\hline \multicolumn{13}{|c|}{ DENSIDADE MINERAL ÓSSEA (mmAl) } \\
\hline \multirow{2}{*}{ Animal } & \multicolumn{4}{|c|}{$\mathrm{C}$} & \multicolumn{4}{|c|}{$\mathrm{S}_{15}$} & \multicolumn{4}{|c|}{$\mathrm{S}_{36}$} \\
\hline & $\mathrm{C} 3$ & T6 & $\mathrm{L} 1$ & L3 & $\mathrm{C} 3$ & T6 & $\mathrm{L} 1$ & L3 & $\mathrm{C} 3$ & T6 & L1 & L3 \\
\hline 1 & 0,89 & 0,82 & 1,43 & 1,66 & 1,06 & 1,15 & 1,77 & 2,14 & 0,93 & 1,00 & 1,48 & 1,73 \\
\hline 2 & 0,76 & 1,03 & 1,89 & 2,01 & 1,24 & 1,11 & 2,23 & 2,68 & 0,83 & 1,08 & 1,94 & 2,24 \\
\hline 3 & 0,79 & 1,10 & * & 1,85 & 1,09 & 1,21 & 2,20 & 2,44 & 0,80 & 1,08 & * & 1,95 \\
\hline 4 & 1,21 & 1,17 & 1,81 & 2,34 & 1,18 & 1,05 & 2,34 & 2,64 & 1,23 & 1,00 & 1,87 & 2,52 \\
\hline 5 & 1,27 & 0,94 & 1,77 & 2,00 & 1,16 & * & 2,27 & 2,69 & 1,15 & 1,00 & 1,93 & 1,91 \\
\hline 6 & 0,91 & 0,84 & 1,42 & 1,60 & 1,08 & 1,12 & 2,48 & 2,53 & 0,93 & 0,80 & 1,46 & 1,71 \\
\hline 7 & 1,07 & 0,78 & 1,55 & 1,99 & 1,16 & 1,00 & 2,33 & 2,34 & 1,21 & 0,94 & 1,66 & 1,95 \\
\hline 8 & 1,03 & 0,84 & 1,57 & 1,81 & 1,07 & 1,12 & 2,54 & 2,70 & 1,07 & 0,79 & 1,66 & 1,88 \\
\hline 9 & 1,10 & 1,06 & 1,65 & 2,04 & 0,88 & 0,91 & 1,87 & 1,98 & 1,17 & 0,95 & 1,64 & 1,90 \\
\hline 10 & 1,40 & 1,16 & 1,76 & 2,41 & 1,18 & 1,18 & 2,38 & 2,66 & 1,36 & 1,18 & 1,37 & 2,38 \\
\hline Média & 1,04 & 0,97 & 1,65 & 1,97 & 1,11 & 1,10 & 2,24 & 2,48 & 1,07 & 0,98 & 1,67 & 2,02 \\
\hline DP & 0,21 & 0,15 & 0,17 & 0,26 & 0,10 & 0,09 & 0,25 & 0,25 & 0,19 & 0,12 & 0,21 & 0,27 \\
\hline $\mathrm{CV}$ & 20,1 & 15,2 & 10,2 & 13,2 & 9,2 & 8,6 & 11,0 & 10,2 & 17,5 & 12,5 & 12,5 & 13,4 \\
\hline
\end{tabular}

*dados perdidos

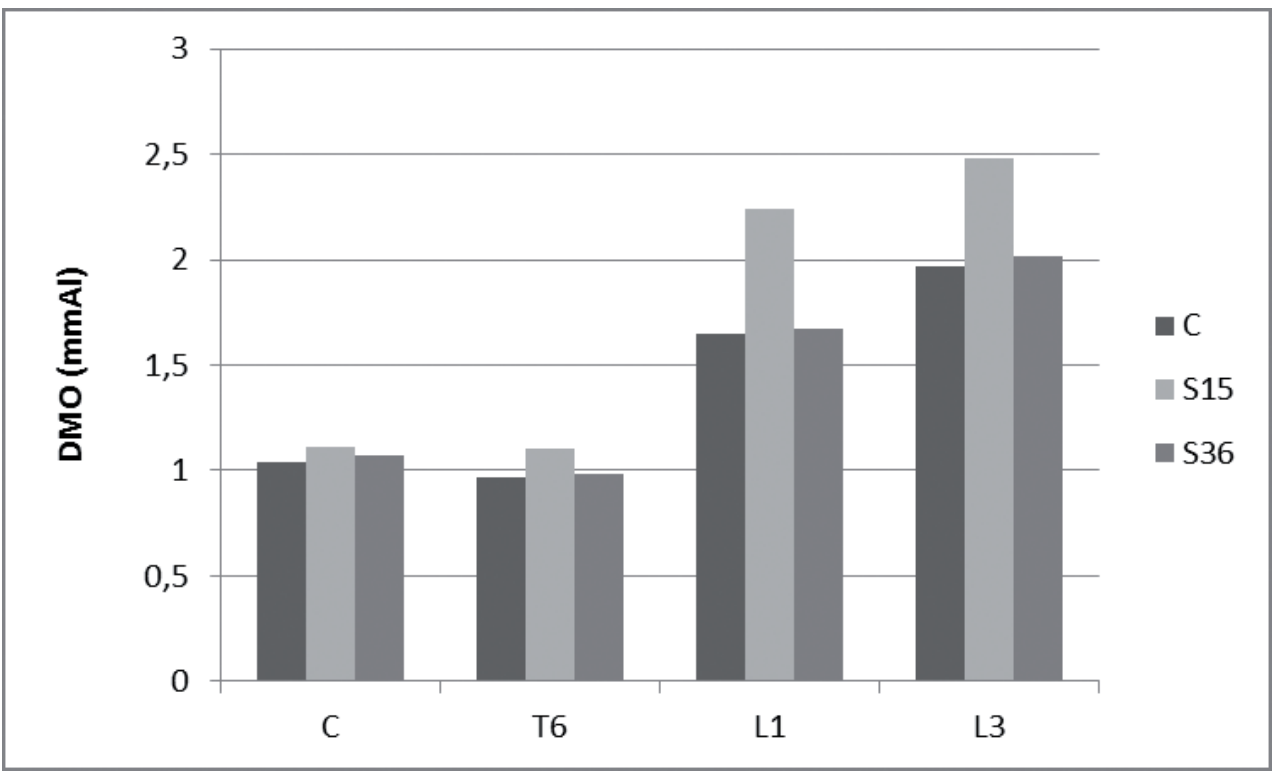

Figura 2 - DMO das vértebras C3, T6, L1 e L3 dos grupos controle; suspenso pela cauda por 15 dias e suspenso pela cauda por 36 dias 


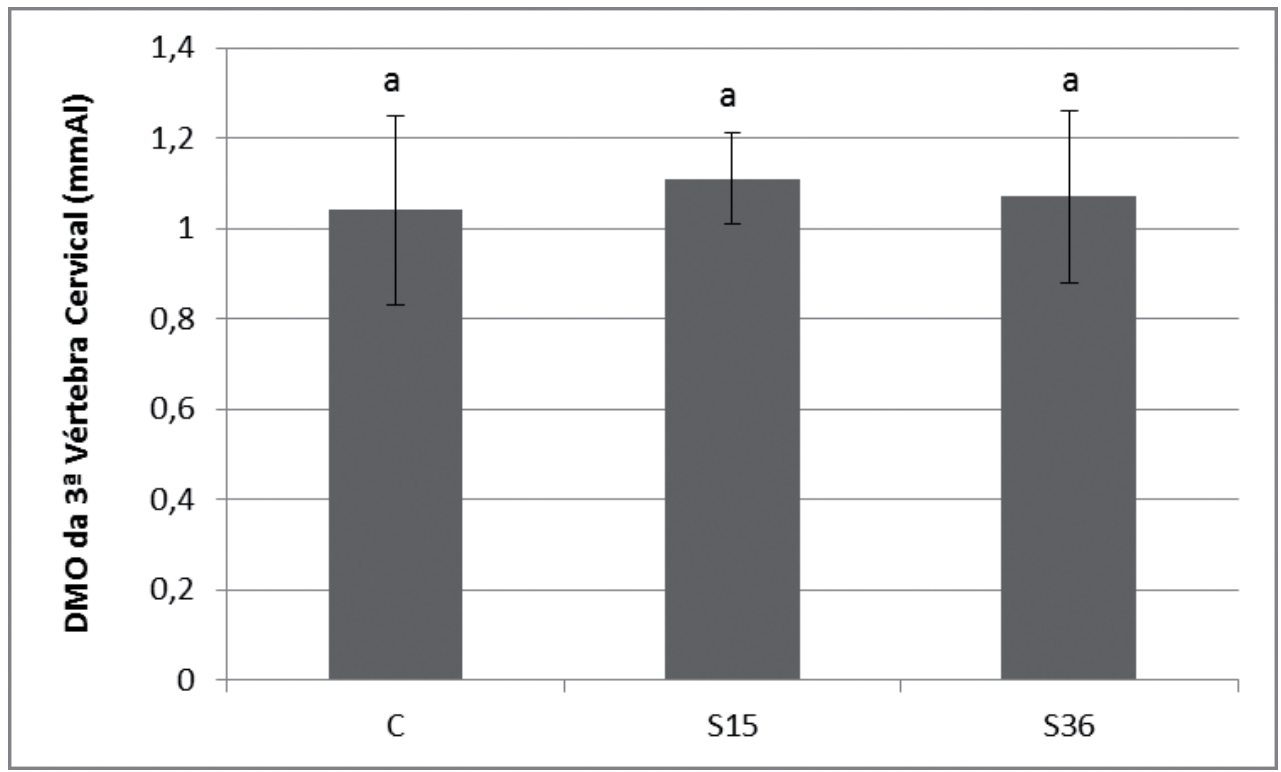

Figura 3 - Comparação da Densidade Mineral Óssea (DMO) da $3^{\text {a }}$ vértebra cervical (mmAl) entre os grupos Controle (C), Suspenso por 15 dias (S15) e Suspenso por 36 dias (S36). Letras iguais implicam em ausência de diferença significativa $(p>0,05)$

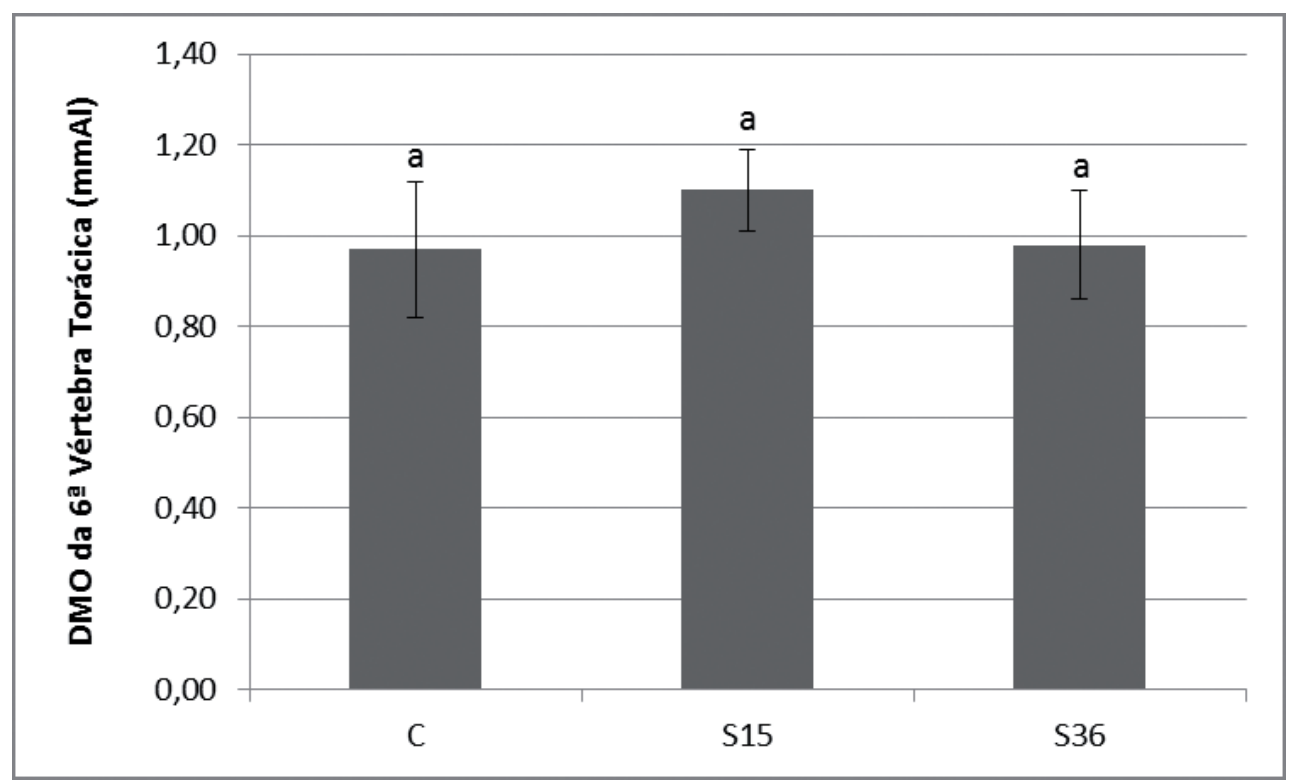

Figura 4 - Comparação da Densidade Mineral Óssea (DMO) da 6 vértebra torácica (mmAl) entre os grupos Controle (C), Suspenso por 15 dias (S15) e Suspenso por 36 dias (S36). Letras iguais implicam em ausência de diferença significativa $(\mathrm{p}>0,05)$ 


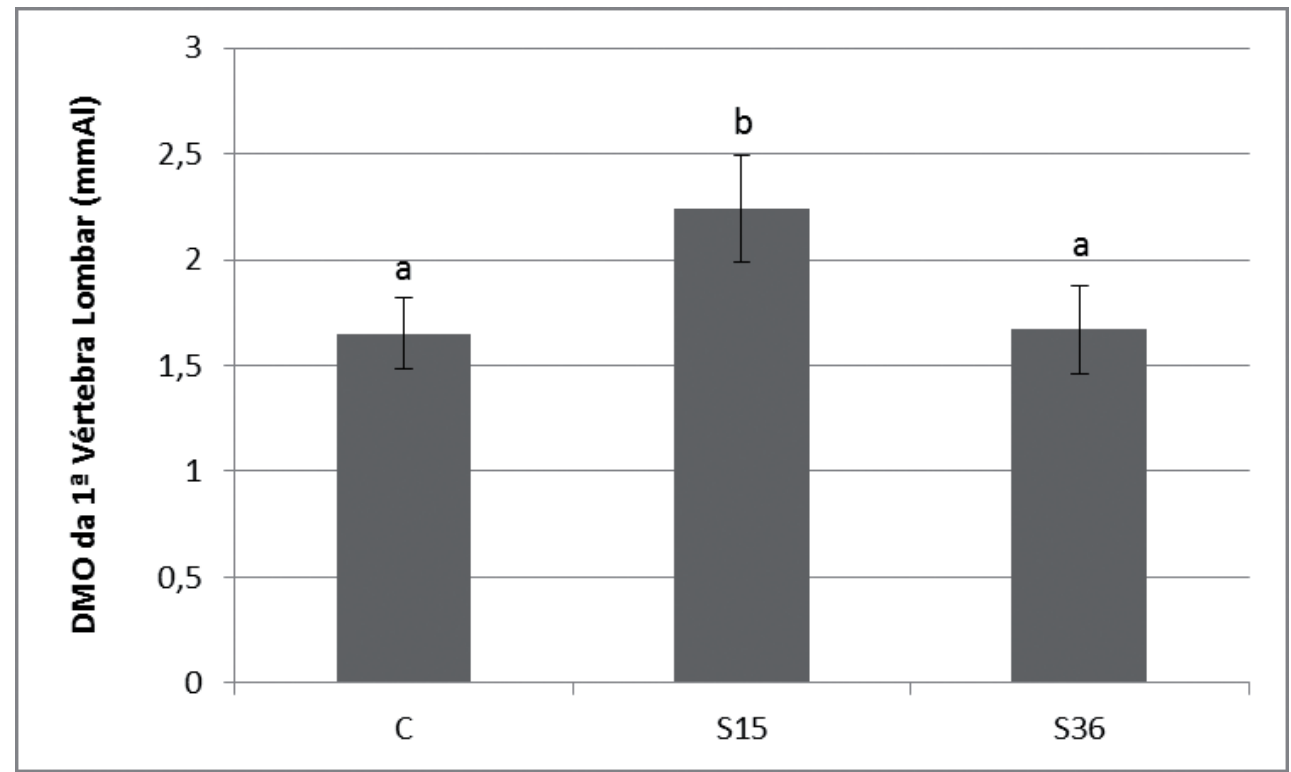

Figura 5 - Comparação da Densidade Mineral Óssea (DMO) da 1a vértebra lombar (mmAl) entre os grupos Controle (C), Suspenso por 15 dias (S15) e Suspenso por 36 dias (S36). Letras diferentes implicam em diferença significativa $(\mathrm{p}<0,05)$

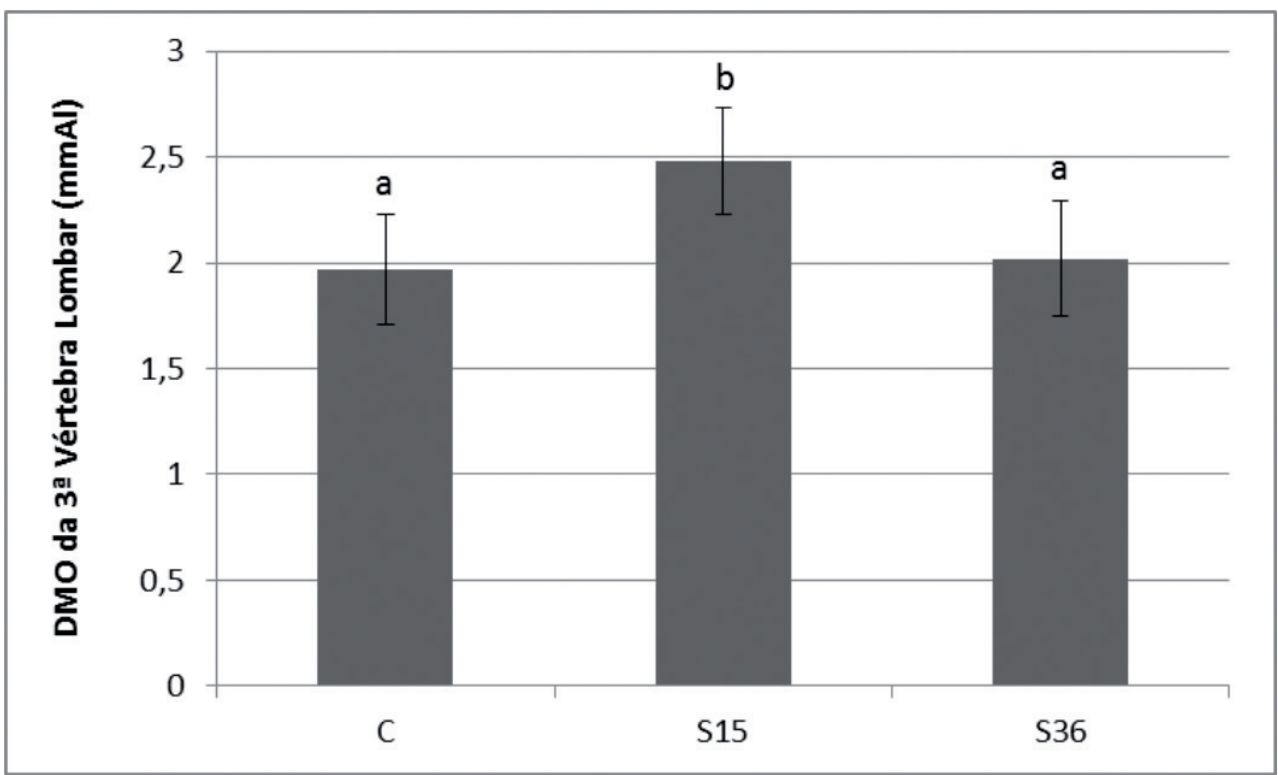

Figura 6 - Comparação da Densidade Mineral Óssea (DMO) da $3^{\text {a }}$ vértebra lombar (mmAl) entre os grupos Controle (C), Suspenso por 15 dias (S15) e Suspenso por 36 dias (S36). Letras diferentes implicam em diferença significativa $(\mathrm{p}<0,05)$ 
mineral ósseo normal depende de solicitações mecânicas geradas também pelas contrações musculares, era esperado que, ao término do experimento, os valores de densidade mineral óssea das vértebras do grupo $S_{36}$ fossem ainda maiores que o do grupo $\mathrm{S}_{15}$. Quando analisados, os valores apresentaram uma regressão muito próxima dos valores do grupo C. Mais uma vez, a ordem dos valores de densidade mineral óssea das vértebras permaneceu na sequência $\mathrm{L} 3>\mathrm{L} 1>\mathrm{C} 3>\mathrm{T} 6$, porém, com valores muitos próximos ao do grupo $\mathrm{C}$, como citado anteriormente. Este fato pode, hipoteticamente, ser atribuído à desistência e cansaço dos animais de se livrarem da

\section{Referências}

1. MUSACCHIA, X. J.; STEFFEN, J. M.; DEAVERS, D. R. Rat hindlimb muscle response to suspension hypokinesia/ hipodynamia. Aviation, Space and Enviromental Medicine, v. 54, n. 11, p. 1015-1020, 1983.

2. KASPER, C. E.; McNULTY, A. L.; OTTO, A. J.; THOMAS, D. P. Alterations in skeletal muscle related to impaired physical mobility: An empirical model. Research in Nursing \& health, v. 16, n. 4, p. 265-273, 1993.

3. SILVA, A. V.; VOLPON, J. B. Modelo de suspensão pela cauda e seu efeito em algumas propriedades mecânicas do osso do rato. Acta Ortopédica Brasileira, v. 12, n. 1, p. 22-31, 2004.

4. VICENTINI, C. R.; ROSA, R. A. C.; CIARLINI, L. D. R. P.; SANTOS, P. H.; LOUZADA, M. J. Q. Análise biomecânica em fêmures de ratos submetidos à ausência de carga e atividade física em esteira. Veterinária e Zootecnia, v. 14, n. 1, p. 62-71, 2007.

5. VICO, L. Summary of research issues in biomechanics and mechanical sensing. Bone, v. 22, n. 5, p. 135-137, 1998.

6. CARVAlho, D. C. L.; ROSIM, G. C.; GAMA, L. O. R.; TAVARES, M. R.; TRIBIOLI, R. A.; SANTOS, I. R.; CLIQUET JR., A. Tratamentos não farmacológicos na estimulação da osteogênese. Revista de Saúde Pública, v. 36, n. 5, p. 647-654, 2002.

7. LOUZADA, M. J. Q. Densitometria radiográfica. 2009. 65 f. Tese (Livre Docência) - Faculdade de Odontologia, Curso de Medicina Veterinária, Universidade Estadual Paulista, Araçatuba, 2009.

8. TURNER, C. H. The biomechanics of hip fracture. Lancet, v. 366, n. 9, p. 98-99, 2005.

9. MAYHEW, P. M.; THOMAS, C. D.; CLEMENT, J. G.; LOVERIDGE, N.; BECK, T. J.; BONFIELD, W.; BURGOYNE, C. J.; REEVE, J. Relation between age, femoral neck, cortical stability and hip fracture risk. Lancet, v. 366, n. 9480, p. 129$135,2005$.

10.AUAD, M. A.; SIMÕES, R. P.; ROUHANI, S.; CASTELO, V.; YOGI, L. S. Eficácia de um programa de exercícios físicos na qualidade de vida de mulheres com osteoporose. Revista suspensão e, por fim, acomodarem-se à situação, diminuindo desta forma, as contrações musculares e não transferindo às vértebras a estimulação necessária para causar deposição óssea, mas sim, ausência na deposição, levando os valores a regredirem muito próximos dos valores do grupo C.

\section{Conclusão}

O estudo demonstrou, pela densitometria radiográfica, que a suspensão de ratos pela cauda causou aumento estatisticamente significante da densidade mineral óssea das vértebras em um primeiro momento, com diminuição com o passar do tempo.

Arquivos de Ciências de Saúde, Santo André, v. 33, n. 1, p. 31-35, 2008.

11.BARBOSA, A. A.; DEL CARMO, R. J.; GALVÃO, S. R.; VILELA, M. J.; LOUZADA, M. J. Q.; BRITO, A. S. F.; NATAL, A. J. Bone mineral densityof rat femurs after hindlimb unloading and diferente physical rehabilition programs. Revista Ceres, Viçosa, v. 58, n. 4, p. 407-412, 2011.

12. RISO, N. D. M. Influência do laser terapêutico no reparo de defeito ósseo de ratos submetidos à ausência de carga: análise densitométrica. 2008. 79 f. Dissertação (Mestrado) Faculdade de Odontologia, Curso de Medicina Veterinária, Universidade Estadual Paulista, Araçatuba, 2008.

13.LOUZADA, M. J. Q.; PELÁ, C. A.; BELANGERO, W. D.; SANTOS-PINTO, R. Metodologia para avaliação de densidade em imagens radiográficas. RBE - Caderno de Engenharia Biomédica, v. 14, n. 2, p. 37-47, 1998.

14.LOUZADA, M. J. Q.; BELANGERO, W. D.; PELÁ, C. A.; SANTOS-PINTO, R. Avaliações de densidade óssea em imagens radiográficas: estudo em peças ósseas de cães. RBE - Caderno de Engenharia Biomédica, v. 14, n. 1, p. 47-64, 1998.

15. APOLINÁRIO, J. C.; COELHO, W. M. D.; LOUZADA, M. J. Q. Análise da influência do ultrassom de baixa intensidade na região de reparo ósseo em ratos sob ausência de carga. Fisioterapia e Pesquisa, v. 18, n. 3, p. 275-279, 2011.

16. HOLICK, M. F. Perspective on the impact of weightlessness on calcium and bone metabolism. Bone, v. 22, p. 105-111, 1998.

17.SHIMANO, M. M. Microestruturas e propriedades mecânicas de ossos cortical e trabecular de ratos após período de suspensão pela cauda e exercitação. 2006. 154 p. Tese (Doutorado) - Faculdade de Medicina, Universidade de São Paulo, Ribeirão Preto, 2006.

18.JOHNSON, R.B. The bearable lightness of being: bones, muscles, and spaceflight. Anatomical Record, v. 253, n. 1, p. 24-27, 1998.

19.GREENE, E. C. Anatomy of the rat. New York: Hafner Publishing Co, 1955. 369 p. 